\title{
Approximate Solutions of Fractional Biological Population Model by Homotopy Analysis Sumudu Transform Method
}

\author{
V. G. Gupta ${ }^{1}$, Pramod Kumar ${ }^{2}$ \\ ${ }^{1,2}$ Department of Mathematics, University of Rajasthan, Jaipur-302004, India
}

\begin{abstract}
In this paper, the Homotopy Analysis Sumudu transform method is presented to find the exact solution of a more general biological population model. The homotopy analysis Sumudu transform method is a combined form of Sumudu transform and homotopy analysis method. The fractional derivatives are described in Caputo sense, some examples are provided.
\end{abstract}

Keywords: Fractional calculus, Sumudu transforms, homotopy analysis method, homotopy analysis Sumudu transforms method, biological population model

\section{Introduction}

In recent year's fractional calculus have been given considerable popularity due mainly to its various applications in fluid mechanics, visco-elasticity, biology, electrical network, optics and signal processing and so on. Except in a limited number of these problems, we have difficulty to find their exact analytic solutions. An effective an easy to use method for solving such equations is needed. Various powerful methods such as differential transform method [1-3], Adomian decomposition method [4-8], Variational iteration method [9-11], homotopy perturbation method [12-16], homotopy perturbation transforms method [17-18] etc., have been proposed to obtain the exact and approximate analytic solutions of fractional differential equations. Another analytical approach that can be applied to solve many types of nonlinear fractional differential equation is Homotopy analysis method (HAM)[19-27). A systematic and clear exposition on HAM is given in [23-24].

The objective of present paper is to apply the homotopy analysis Sumudu transform method, which is an elegant combination of Sumudu transform method and homotopy analysis method, to find the solution of time-fractional biological population model [28], a representative biological population diffusion equation is $u_{t}=u_{x x}^{2}+u_{y y}^{2}+f(u)$, where $u(x, y, t)$ denotes the population density and $f(u)$ represents the population supply due to birth and death. In this paper, we propose a generalized time-fractional nonlinear biological population diffusion equation as follows:

$$
\frac{\partial^{\alpha} u}{\partial t^{\alpha}}=\frac{\partial^{2} u^{2}}{\partial x^{2}}+\frac{\partial^{2} u^{2}}{\partial y^{2}}+f(u), t>0, \quad x, y \in R
$$

with given initial conditions $u(x, y, 0)$, and according to Malthusian law and Verhulst law, we consider a more general form of $f(x)=h u^{a}\left(1-r u^{b}\right)$, where $h, a, b, r$ are real numbers. When choose special values, they change to Malthusian law and Verhulst law.

The derivatives in Eq. (1.1) are the Caputo derivative. Linear and Nonlinear population systems were solved by using
Variational iteration method [28], Adomian Decomposition method [29], and Homotopy perturbation method [30]. However, one of the disadvantages ADM is the inherent difficulty in calculating the Adomian polynomial. This paper considers the effectiveness of the homotopy analysis Sumudu transform method (HASTM) in solving fractional biological population system.

\section{Basic Definitions}

For the concept of fractional derivatives, we will adopt Caputo's definition which is a modification of the Riemann-Liouville definition and has the advantage of dealing properly with initial value problems in which the initial conditions are given in terms of the field variable and their integral order which is the case in most physical processes. Some basic definitions and properties of fractional calculus theory which we have used in this paper are given in this section.

Definition 2.1 A real function $\mathrm{f}(\mathrm{x}), \mathrm{x}>0$ is said to be in the space $C_{\mu}, \mu \in R$, if there exist a real number $p(>\mu)$ such that $f(x)=x^{p} f_{1}(x)$, where $f_{1}(x) \in C[0, \infty)$, and it is said to be in the space $\mathrm{C}_{\mu}^{\mathrm{m}}$ iff $\mathrm{f}^{(\mathrm{m})} \in \mathrm{C}_{\mu}, \mathrm{m} \in \mathrm{N} \cup\{0\}$.

Definition 2.2.The Riemann-Liouville fractional integral operator of order $\alpha \geq 0$ of a function $\mathrm{f} \in \mathrm{C}_{\mu}, \mu \geq-1$ is defined as

$$
\begin{gathered}
\mathrm{J}^{\alpha} \mathrm{f}(\mathrm{x})=\frac{1}{\Gamma \alpha} \int_{0}^{\mathrm{x}}(\mathrm{x}-\mathrm{t})^{\alpha-1} \mathrm{f}(\mathrm{t}) \mathrm{dt}, \quad \alpha>0, \mathrm{x}>0 \\
\mathrm{~J}^{0} \mathrm{f}(\mathrm{x})=\mathrm{f}(\mathrm{x})
\end{gathered}
$$

Properties of the operator $\mathbf{J}^{\alpha}$ can be found in [31], we mention only the following:
(i) $\mathrm{J}^{\alpha} \mathrm{J}^{\beta} \mathrm{f}(\mathrm{x})=\mathrm{J}^{\alpha+\beta} \mathrm{f}(\mathrm{x})$
(ii) $\mathrm{J}^{\alpha} \mathrm{J}^{\beta} \mathrm{f}(\mathrm{x})=\mathrm{J}^{\beta} \mathrm{J}^{\alpha} \mathrm{f}(\mathrm{x})$ 


\section{International Journal of Science and Research (IJSR) \\ ISSN (Online): 2319-7064}

Index Copernicus Value (2013): 6.14 | Impact Factor (2015): 6.391

(iii) $\mathrm{J}^{\alpha} \mathrm{x}^{\gamma}=\frac{\Gamma(\gamma+1)}{\Gamma(\alpha+\gamma+1)} \mathrm{x}^{\alpha+\gamma}$

For $\mathrm{f} \in \mathrm{C}_{\mu}, \mu \geq-1, \alpha, \beta \geq 0$ and $\gamma>-1$.

Definition 2.3.The fractional derivative of $f(x)$ in the Caputo sense is defined as [31]

$$
\mathrm{D}_{t}^{\alpha} \mathrm{f}(\mathrm{x})=\mathrm{J}^{\mathrm{m}-\alpha} \mathrm{D}_{*}^{\mathrm{m}} \mathrm{f}(\mathrm{x})=\frac{1}{\Gamma(\mathrm{m}-\alpha)} \int_{0}^{\mathrm{x}}(\mathrm{x}-\mathrm{t})^{\mathrm{m}-\alpha-1} \mathrm{f}^{(\mathrm{m})}(\mathrm{t}) \mathrm{dt}
$$

For $\mathrm{m}-1<\alpha \leq \mathrm{m}, \mathrm{m} \in \mathrm{N}, \mathrm{x}>0, \mathrm{f} \in \mathrm{C}_{-1}^{\mathrm{m}}$.

Also, we need here three basic properties

(i) $\mathrm{D}_{t}^{\alpha} \mathrm{J}^{\alpha} \mathrm{f}(\mathrm{x})=\mathrm{f}(\mathrm{x})$

(ii)

$$
\mathrm{J}^{\alpha} \mathrm{D}_{t}^{\alpha} \mathrm{f}(\mathrm{x})=\mathrm{f}(\mathrm{x})-\sum_{\mathrm{k}=0}^{\mathrm{m}-1} \mathrm{f}^{(\mathrm{k})}\left(0^{+}\right) \frac{\mathrm{x}^{\mathrm{k}}}{\mathrm{k} !}, \quad \mathrm{x}>0
$$

(iii) $\mathrm{D}_{t}^{\alpha} \mathrm{x}^{\gamma}=\frac{\Gamma(\gamma+1)}{\Gamma(\gamma-\alpha+1)} \mathrm{x}^{\gamma-\alpha} ; \mathrm{x}>0, \gamma>0$.

For $\mathrm{m}-1<\alpha \leq \mathrm{m}, \mathrm{m} \in \mathrm{N}, \mu \geq-1$ and $\mathrm{f} \in \mathrm{C}_{\mu}^{\mathrm{m}}$.

Lemma 2.1.If $m-1<\alpha \leq m, m \in N$, then the

Laplace transform of the fractional derivative $D_{t}^{\alpha} f(t)$ is

$\mathrm{L}\left(\mathrm{D}_{t}^{\alpha} \mathrm{f}(\mathrm{t})\right)=\mathrm{s}^{\alpha} \overline{\mathrm{f}}(\mathrm{s})-\sum_{\mathrm{k}=0}^{\mathrm{m}-1} \mathrm{f}^{(\mathrm{k})}\left(0^{+}\right) \mathrm{s}^{\alpha-\mathrm{k}-1}, \mathrm{t}>0$

Where $\bar{f}(s)$ is the Laplace transform of $f(t)$.

\section{Sumudu Transform}

In early 90's Watugala [32], introduced a new integral transform, named the Sumudu transform and applied it to the solution of ordinary differential equation in control engineering problems. The Sumudu transform is defined over the set of functions

$A=\left\{f(t)\left|\exists M, \tau_{1}, \tau_{2}>0,\right| f(t)<M e^{|t| / \tau_{j}}\right.$, if $\left.t \in(-i)^{j} \times[0, \infty)\right\}$

by the following formula

$$
G(u)=S[F(t)]=\int_{0}^{\infty} F(u t) e^{-t} d t, \quad u \in\left(-\tau_{1}, \tau_{2}\right)
$$

The existence and uniqueness of this transformation is discussed in [33]. For further details and properties of this transformation, see [34-36].

\section{Basic Idea of Homotopy Analysis Method}

To give the basic idea of Homotopy Analysis Method [23], let us consider a nonlinear differential equation in the form:

$$
\square[u(x, t)]=0 \quad t \geq 0
$$

Where $\square$ is a fractional differential operator and $\mathrm{u}(\mathrm{x}, t)$ is unknown function of the independent variable $x$ and $t$. For the simplicity we ignore all boundary or initial conditions, which can be treated in the similar way.

In the frame of HAM [22-23], we can construct the following zeroth-order deformation equation:

$$
(1-q) L\left[\phi(x, t ; q)-u_{0}(x, t)\right]=q \hbar H(t) \square[\phi(x, t ; q)]
$$

Where $q \in[0,1]$ is an embedding parameter, $h \neq 0$ is an auxiliary parameter, $H(t) \neq 0$ is an auxiliary function, $L$ is an auxiliary linear operator, $\square$ is fractional differential operator, $\phi(x, t ; q)$ is an unknown function, and $u_{0}(t)$ is an initial guess of $u(x, t)$, which satisfies the initial conditions. It should be emphasized that one has great freedom to choose the initial guess $u_{0}(t)$, the auxiliary linear operator $L$, the auxiliary parameter $\hbar$ and the auxiliary function $H(t)$. Obviously, when the embedding parameter $q=0$ and $q=1$, it holds

$$
\phi(x, t ; 0)=u_{0}(x, t), \phi(x, t ; 1)=u(x, t)
$$

respectively. Thus as $q$ increases from 0 to 1 , the solution $\phi(x, t ; q)$ varies from the initial guess $u_{0}(x, t)$ to $u(x, t)$ . Expending $\phi(x, t ; q)$ in Taylor series with respect to $q$, we have

$$
\phi(x, t ; q)=u_{0}(x, t)+\sum_{m=1}^{\infty} u_{m}(x, t) q^{m}
$$

where

$$
u_{m}(x, t)=\left.\frac{1}{m !} \frac{\partial^{m} \phi(x, t ; q)}{\partial q^{m}}\right|_{q=0}
$$

Assume that the auxiliary parameter, the auxiliary function $H(t)$, the initial approximation and the auxiliary linear operator $L$ are properly chosen, the series (4.3) converges at $q=1$, then we have

$$
u(x, t)=u_{0}(x, t)+\sum_{m=1}^{\infty} u_{m}(x, t)
$$

which must be one of the solution of the original nonlinear equations. According to the definition (4.5), the governing equation can be deduced from the zero-order deformation (4.2). Define the vectors

$$
\vec{u}_{m}=\left\{u_{0}(x, t), u_{1}(x, t), u_{2}(x, t), \ldots, u_{n}(x, t)\right\}
$$

Differentiating equation (4.2), m-times with respect to embedding parameter $\mathrm{q}$, then setting $\mathrm{q}=0$ and dividing them by $m$ ! , we get the so-called mth-order deformation equation

$$
L\left[u_{m}(x, t)-\chi_{m} u_{m-1}(x, t)\right]=\hbar H(t) \mathfrak{R}_{m}\left(\vec{u}_{m-1}\right)
$$

Where

$$
\mathfrak{R}_{m}\left(\vec{u}_{m-1}\right)=\left.\frac{1}{(m-1) !} \frac{\partial^{m-1} \square[\phi(x, t ; q)]}{\partial q^{m-1}}\right|_{q=0}
$$

and

$$
\chi_{m}= \begin{cases}0, & m \leq 1 \\ 1, & m>1\end{cases}
$$

The so-called mth-order deformation equation (4.7) is linear which can be easily solved using Mathematica package. 


\section{International Journal of Science and Research (IJSR) \\ ISSN (Online): 2319-7064}

Index Copernicus Value (2013): 6.14 | Impact Factor (2015): 6.391

\section{Homotopy Analysis Sumudu Transform Method}

To illustrate the basic idea of this method, let us consider a general fractional nonlinear non homogeneous differential equation

$$
D_{t}^{\alpha} u(x, t)+R u(x, t)+N u(x, t)=g(x, t), 0<\alpha \leq 1
$$

Where $g(x, t)$ is the source term, $N$ represent the general nonlinear differential operator and $\mathrm{R}$ is the linear differential operator, $D_{t}^{\alpha}$ is the Caputo fractional derivative of the function $u(x, t)$.

Now taking the Sumudu transform of both sides of (5.1), we get

$$
S\left[D_{t}^{\alpha} u(x, y, t)\right]+S[R(u, t)]+S[N u(x, t)]=S[g(x)]
$$

Subject to the initial conditions

$$
u(x, 0)=f(x)
$$

Using the differentiation properties of the Sumudu transform and above initial condition, we have

$$
\begin{array}{r}
\frac{S[u]}{u^{\alpha}}-\sum_{k=0}^{n-1} \frac{u^{(k)}(0)}{u^{n-k}}+S[R u(x, t)]+S[N u(x, t)]=S[g(x)] \\
S[u(x, t)]-u^{\alpha} \sum_{k=0}^{n-1} \frac{u^{(k)}(0)}{u^{n-k}}+u^{\alpha}[S[R u(x, t)]+S[N u(x, t)]-S[g(x)]]
\end{array}
$$

We define the nonlinear operator

$$
N[\phi(x, t ; q)]=S[\phi(x, t ; q)]-u^{\alpha} \sum_{k=0}^{n-1} \frac{\phi^{(k)}(0)}{u^{n-k}}+u^{\alpha}[S[R \phi(x, t ; q)]+S[N \phi(x, t ; q)]-S[g(x)]]
$$

Where $q \in[0,1]$ and $\phi(x, t ; q)$ is a real function of $x, t, q$.

The so-called zero-order deformation equation of the Eq. (5.6) has the form

$$
(1-q) S\left[\phi(x, t ; q)-u_{0}(x, t)\right]=q \hbar H(x, t)\left[S[\phi(x, t ; q)]-f(x)-u^{\alpha} S[R \phi(x, t ; q)+N \phi(x, t ; q)-g(x, t)]\right]
$$

Where $\mathrm{S}$ is the Sumudu transform, $q \in[0,1]$ is the embedding parameter, $H(x, t)$ denotes a nonzero auxiliary function, $\hbar \neq 0$ is an auxiliary parameter, $u_{0}(x, t)$ is an initial guess of $u(x, t)$ and $\phi(x, t ; q)$ is an unknown function. Obviously, when the parameter $\mathrm{q}=0$ and $\mathrm{q}=1$, it holds

$$
\phi(x, t ; 0)=u_{0}(x, t) \quad, \phi(x, t ; 1)=u(x, t)(5.8)
$$

respectively. Thus as $\mathrm{q}$ increases from 0 to 1 , the solution $\phi(x, t ; q)$ varies from the initial guess $u_{0}(x, t)$ to the solution $u(x, t)$. Expanding $\phi(x, t ; q)$ in Taylor series with respect to $\mathrm{q}$, we have

$$
\phi(x, t ; q)=u_{0}(x, t)+\sum_{m=1}^{\infty} u(x, t) q^{m}(5.9) \quad \text { and }
$$

Where

$$
u_{m}(x, t)=\left.\frac{1}{m !} \frac{\partial^{m} \phi(x, t ; q)}{\partial q^{m}}\right|_{q=0}
$$

If the auxiliary linear operator, the initial guess, the auxiliary parameter $\hbar$, and the auxiliary function are properly chosen, the series (5.9) converges at $q=1$, then we has

$$
u(x, t)=u_{0}(x, t)+\sum_{m=1}^{\infty} u_{m}(x, t)
$$

Which must be one of the solution of the original nonlinear equations. According to the definition (5.11), the governing equation can be deduced from the zero- order deformation (5.7). Define the vectors

$$
\vec{u}(x, t)=\left\{u_{0}(x, t), u_{1}(x, t), u_{2}(x, t), \ldots, u_{m}(x, t)\right\}
$$

Differentiating the zero- order deformation equation (5.7) mtimes with respect to $\mathrm{q}$ and then dividing by $m$ ! and finally setting $\mathrm{q}=0$ we get the following $\mathrm{m}^{\text {th }}$ - order deformation equation:

$$
S\left[u_{m}(x, t)-\chi_{m} u_{m-1}(x, t)\right]=\hbar H(x, t) \mathfrak{R}_{m}\left(\vec{u}_{m-1}(x, t)\right)
$$

Applying the inverse Sumudu transform, we have $\left.u_{m}(x, t)=\chi_{m} u_{m-1}(x, t)\right]+S^{-1}\left[\hbar H(x, t) \mathfrak{R}_{m}\left(\vec{u}_{m-1}(x, t)\right)\right]$

where

$$
\mathfrak{R}_{m}\left(\vec{u}_{m-1}\right)=\left.\frac{1}{(m-1) !} \frac{\partial^{m-1} N[\phi(x, t ; q)]}{\partial q^{m-1}}\right|_{q=0}
$$

$$
\chi_{m}=\left\{\begin{array}{l}
0, m \leq 1 \\
1, m>1
\end{array}\right.
$$

\section{Numerical Results}

In this section we use the Homotopy Analysis Sumudu transform method to solve nonlinear fractional biological population equations:

Example1. Consider the Eq.(1.1) with $a=1, \quad r=0$, corresponding to Malthusian law, we have the following biological population equation

$$
\frac{\partial^{\alpha} u}{\partial t^{\alpha}}=\frac{\partial^{2} u^{2}}{\partial x^{2}}+\frac{\partial^{2} u^{2}}{\partial y^{2}}+h u
$$

Subject to the initial condition 


\section{International Journal of Science and Research (IJSR) \\ ISSN (Online): 2319-7064}

Index Copernicus Value (2013): 6.14 | Impact Factor (2015): 6.391

$$
u_{0}=\sqrt{x y}
$$

Taking the Sumudu transform of both sides of Eq. (6.1), and using (6.2)

$$
S[u]-\sqrt{x y}-u^{\alpha}\left[S\left[\frac{\partial^{2} u^{2}}{\partial x^{2}}+\frac{\partial^{2} u^{2}}{\partial y^{2}}+h u\right]\right]=0
$$

Then

$$
\mathfrak{R}\left(\bar{u}_{m-1}\right)=S\left[u_{m-1}\right]-\left(1-\chi_{m}\right) \sqrt{x y}-u^{\alpha}\left[S\left[\frac{\partial^{2}}{\partial x^{2}} \sum_{j=0}^{m-1} u_{j} u_{m-1-j}\right]+S\left[\frac{\partial^{2}}{\partial y^{2}} \sum_{j=0}^{m-1} u_{j} u_{m-1-j}\right]+S\left[h u_{m-1}\right]\right]
$$

The $\mathrm{m}^{\text {th }}$ - order deformation is given by

$$
S\left[u_{m}(x, t)-\chi_{m} u_{m-1}(x, t)\right]=\hbar \Re_{m}\left(\vec{u}_{m-1}\right)
$$

Applying the inverse Sumudu transform, we have

$$
u_{m}(x, t)=\chi_{m} u_{m-1}(x, t)+S^{-1}\left[\hbar \Re_{m}\left(\vec{u}_{m-1}\right)\right]
$$

Solving eq. (6.6) for $\mathrm{m}=1,2,3, \ldots$ we have

$$
\begin{aligned}
& u_{1}(x, y, t)=-h \hbar \sqrt{x y} \frac{t^{\alpha}}{\Gamma(1+\alpha)} \\
& u_{2}(x, y, t)=-h \hbar(1+\hbar) \sqrt{x y} \frac{t^{\alpha}}{\Gamma(1+\alpha)}+h^{2} \hbar^{2} \sqrt{x y} \frac{t^{2 \alpha}}{\Gamma(1+2 \alpha)} \\
& u_{3}(x, y, t)=-h \hbar(1+\hbar)^{2} \sqrt{x y} \frac{t^{\alpha}}{\Gamma(1+\alpha)}+2 h^{2} \hbar^{2}(1+\hbar) \sqrt{x y} \frac{t^{2 \alpha}}{\Gamma(1+2 \alpha)}-h^{3} \hbar^{3} \sqrt{x y} \frac{t^{3 \alpha}}{\Gamma(1+3 \alpha)} \\
& u_{4}(x, y, t)=-h \hbar(1+\hbar)^{3} \sqrt{x y} \frac{t^{\alpha}}{\Gamma(1+\alpha)}+3 h^{2} \hbar^{2}(1+\hbar)^{2} \sqrt{x y} \frac{t^{2 \alpha}}{\Gamma(1+2 \alpha)} \\
& -3 h^{3} \hbar^{3}(1+\hbar) \sqrt{x y} \frac{t^{3 \alpha}}{\Gamma(1+3 \alpha)}+h^{4} \hbar^{4} \sqrt{x y} \frac{t^{4 \alpha}}{\Gamma(1+4 \alpha)}
\end{aligned}
$$

substituting $u_{0}, u_{1}, u_{2}, u_{3}, u_{4}, \ldots$, into Eq. (5.11) gives the solution in series form by:

$$
\begin{aligned}
& u(x, y, t)=\sqrt{x y}\left[1-h \hbar\left\{1+(1+\hbar)+(1+\hbar)^{2}+(1+\hbar)^{3}+\ldots\right\} \frac{t^{\alpha}}{\Gamma(1+\alpha)}+\right. \\
& \left.h^{2} \hbar^{2}\left\{1+2(1+\hbar)+3(1+\hbar)^{2}+\ldots\right\} \frac{t^{2 \alpha}}{\Gamma(1+2 \alpha)}-h^{3} \hbar^{3}\{1-3(1+\hbar)+\ldots\} \frac{t^{3 \alpha}}{\Gamma(1+3 \alpha)}+h^{4} \hbar^{4}\{1+\ldots\} \frac{t^{4 \alpha}}{\Gamma(1+4 \alpha)}+\ldots\right]
\end{aligned}
$$

Setting $\hbar=-1$,

$$
u(x, y, t)=\sqrt{x y}\left[1+h \frac{t^{\alpha}}{\Gamma(1+\alpha)}+h^{2} \frac{t^{2 \alpha}}{\Gamma(1+2 \alpha)}+h^{3} \frac{t^{3 \alpha}}{\Gamma(1+3 \alpha)}+h^{4} \frac{t^{4 \alpha}}{\Gamma(1+4 \alpha)}+\ldots\right]
$$

as $\alpha \rightarrow 1$, we have

$$
\begin{gathered}
u(x, y, t)=\sqrt{x y}\left[1+h t+\frac{h^{2} t^{2}}{2 !}+\frac{h^{3} t^{3}}{3 !}+\frac{h^{4} t^{4}}{4 !}+\ldots\right] \\
u(x, y, t)=\sqrt{x y} e^{h t}
\end{gathered}
$$

which is an exact solution to the standard form biological population equation. The evolution result for the exact solution (6.10) and the approximate solution (6.9) for the case $\alpha=1$, are shown in Fig.(1). It can be seen from Fig. 1 that the solutions obtained by the HASTM is nearly identical with the exact solution. Fig.2 show the approximate solutions. It also be concluded that the approximate solution of fractional biological model is continuous with the parameter $\alpha$. 


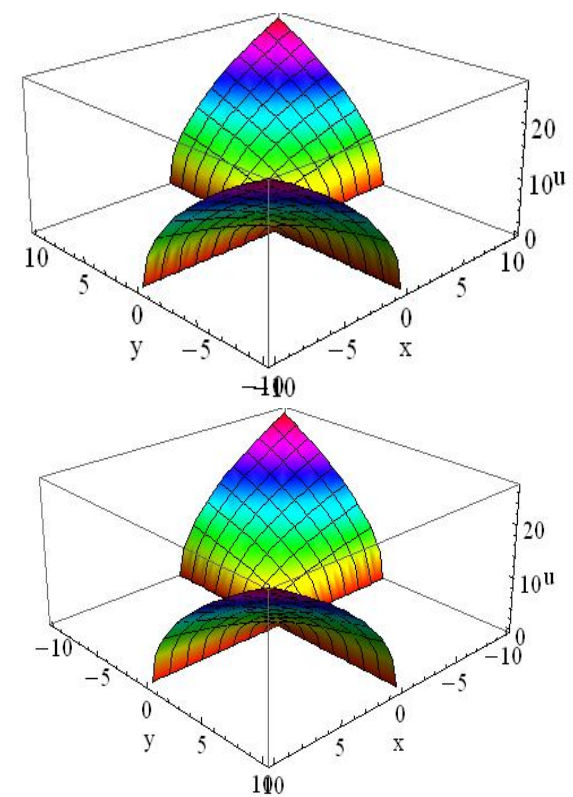

Figure 1: The surface shows the solution $u(x, y, t)$ for $(6.8)$ : (i) exact solution (6.10); (ii) numerical solution (6.9) when $h=0.1, t=10$
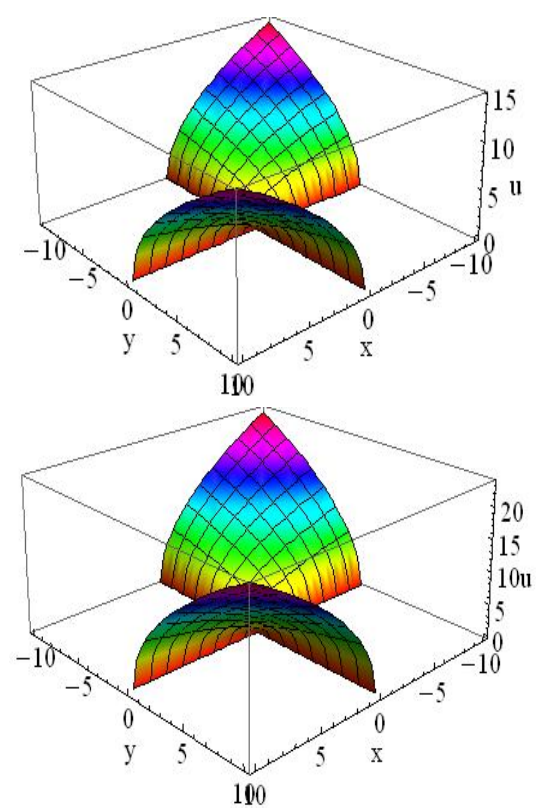

Figure 2: The surface shows the solution $u(x, y, t)$ for (6.7): (i) $\hbar=-1.4, \alpha=0.5$ (ii) $\hbar=-1, \alpha=0.9$ when $h=0.1$, $t=10$

Example2. Consider the Eq. (1.1) with $a=1, b=1$, this leads to Verhulst law, and we have the following fractional biological population equation

$$
\frac{\partial^{\alpha} u}{\partial t^{\alpha}}=\frac{\partial^{2} u^{2}}{\partial x^{2}}+\frac{\partial^{2} u^{2}}{\partial y^{2}}+h u(1-h u)
$$

subject to the initial condition

$$
u_{0}=e^{\sqrt{\frac{h r}{8}}(x+y)}
$$

Taking the Sumudu transform of Eq. (6.11) and using the Eq. (6.12), we have

$$
S[u]-e^{\sqrt{\frac{h r}{8}}(x+y)}-u^{\alpha}\left[S\left[\frac{\partial^{2} u^{2}}{\partial x^{2}}+\frac{\partial^{2} u^{2}}{\partial y^{2}}+\left(h u-h r u^{2}\right)\right]\right]=0
$$

$$
\begin{aligned}
\mathfrak{R}\left(\bar{u}_{m-1}\right)= & \left.\left.S\left[u_{m-1}\right]-\left(1-\chi_{m}\right) e^{\sqrt{\frac{h r}{8}}(x+y)}\right]+S\left[\frac{\partial^{2}}{\partial y^{2}} \sum_{j=0}^{m-1} u_{j} u_{m-1-j}\right]+S\left[h u_{m-1}-h r \sum_{j=0}^{m-1} u_{j} u_{m-1-j}\right]\right] \\
& -u^{\alpha}\left[S\left[\frac{\partial^{2}}{\partial x^{2}} \sum_{j=0}^{m-1} u_{j} u_{m-1-j}\right]+\right.
\end{aligned}
$$

The $\mathrm{m}^{\text {th }}$ - order deformation is given by

$$
S\left[u_{m}(x, t)-\chi_{m} u_{m-1}(x, t)\right]=\hbar \Re_{m}\left(\vec{u}_{m-1}\right)
$$

Applying the inverse Sumudu transform, we have

$$
u_{m}(x, t)=\chi_{m} u_{m-1}(x, t)+S^{-1}\left[\hbar \Re_{m}\left(\vec{u}_{m-1}\right)\right]
$$

Solving eq. (6.16) for $m=1,2,3, \ldots$, we have

$$
\begin{aligned}
& u_{1}=-h \hbar e^{\sqrt{\frac{h r}{8}}(x+y)} \frac{t^{\alpha}}{\Gamma(1+\alpha)} \\
& u_{2}=-h \hbar(1+\hbar) e^{\sqrt{\frac{h r}{8}(x+y)}} \frac{t^{\alpha}}{\Gamma(1+\alpha)}+h^{2} \hbar^{2} e^{\sqrt{\frac{h r}{8}}(x+y)} \frac{t^{2 \alpha}}{\Gamma(1+2 \alpha)} \\
& u_{3}=-h \hbar(1+\hbar)^{2} e^{\sqrt{\frac{h r}{8}}(x+y)} \frac{t^{\alpha}}{\Gamma(1+\alpha)}+2 h^{2} \hbar^{2}(1+\hbar) e^{\sqrt{\frac{h r}{8}}(x+y)} \frac{t^{2 \alpha}}{\Gamma(1+2 \alpha)}-h^{3} \hbar^{3} e^{\sqrt{\frac{h r}{8}}(x+y)} \frac{t^{3 \alpha}}{\Gamma(1+3 \alpha)}
\end{aligned}
$$

\section{Volume 5 Issue 5, May 2016}

$$
\text { www.ijsr.net }
$$




\section{International Journal of Science and Research (IJSR) \\ ISSN (Online): 2319-7064}

Index Copernicus Value (2013): 6.14 | Impact Factor (2015): 6.391

$$
\begin{aligned}
u_{4}=-h \hbar(1+\hbar)^{3} e^{\sqrt{\frac{h r}{8}}(x+y)} \frac{t^{\alpha}}{\Gamma(1+\alpha)}+3 h^{2} \hbar^{2}(1+\hbar)^{2} e^{\sqrt{\frac{h r}{8}}(x+y)} \frac{t^{2 \alpha}}{\Gamma(1+2 \alpha)} \\
-3 h^{3} \hbar^{3}(1+\hbar) e^{\sqrt{\frac{h r}{8}}(x+y)} \frac{t^{3 \alpha}}{\Gamma(1+3 \alpha)}+h^{4} \hbar^{4} e^{\sqrt{\frac{h r}{8}}(x+y)} \frac{t^{4 \alpha}}{\Gamma(1+4 \alpha)}
\end{aligned}
$$

substituting $\mathrm{u}_{0}, \mathrm{u}_{1}, \mathrm{u}_{2}, \mathrm{u}_{3}, \mathrm{u}_{4}, \ldots$, into Eq. (5.11) gives the solution in series form by:

$$
\begin{gathered}
u=e^{\sqrt{\frac{h r}{8}(x+y)}}\left[1-h \hbar\left\{1+(1+\hbar)+(1+\hbar)^{2}+(1+\hbar)^{3}+\ldots\right\} \frac{t^{\alpha}}{\Gamma(1+\alpha)}+h^{2} \hbar^{2}\left\{1+2(1+\hbar)+3(1+\hbar)^{2}+\ldots\right\} \frac{t^{2 \alpha}}{\Gamma(1+2 \alpha)}-\right. \\
\left.h^{3} \hbar^{3}\{1-3(1+\hbar)+\ldots\} \frac{t^{3 \alpha}}{\Gamma(1+3 \alpha)}+h^{4} \hbar^{4}\{1+\ldots\} \frac{t^{4 \alpha}}{\Gamma(1+4 \alpha)}+. .\right]
\end{gathered}
$$

Set $\hbar=-1$,

$$
u(x, y, t)=e^{\sqrt{\frac{h r}{8}}(x+y)}\left[1+h \frac{t^{\alpha}}{\Gamma(1+\alpha)}+h^{2} \frac{t^{2 \alpha}}{\Gamma(1+2 \alpha)}+h^{3} \frac{t^{3 \alpha}}{\Gamma(1+3 \alpha)}+h^{4} \frac{t^{4 \alpha}}{\Gamma(1+4 \alpha)}+\ldots\right]
$$

as $\alpha \rightarrow 1$, we have

$u(x, y, t)=e^{\sqrt{\frac{h r}{t}}(x+y)+h t}(6.20)$

$$
u(x, y, t)=e^{\sqrt{\frac{h r}{8}}(x+y)}\left[1+h t+\frac{h^{2} t^{2}}{2 !}+\frac{h^{3} t^{3}}{3 !}+\frac{h^{4} t^{4}}{4 !}+\ldots\right]
$$

which is an exact solution to the standard form biological model [6.11]

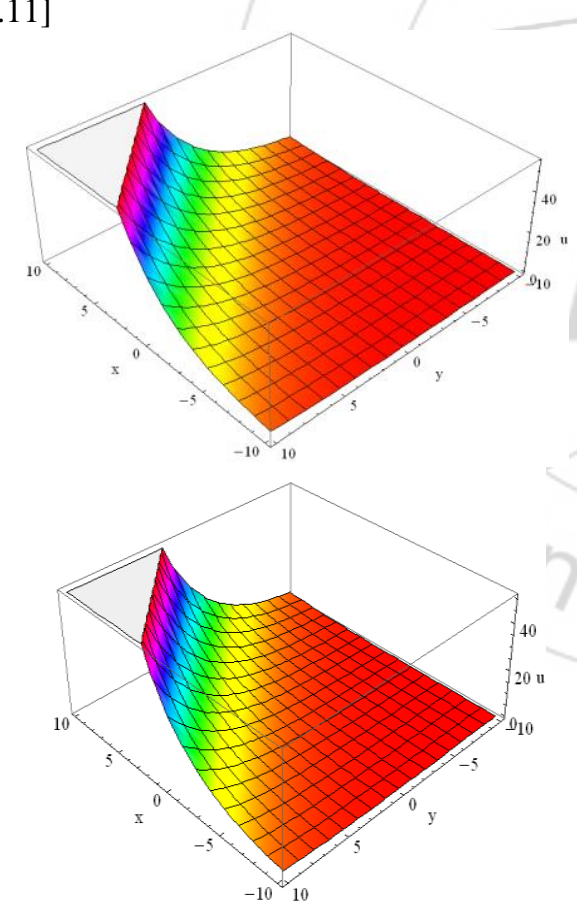

Figure 3: The surface shows the solution $u(x, y, t)$ for (6.18): (i) exact solution (6.20);(ii) numerical solution (6.19) when $h=0.01, t=10, r=48$

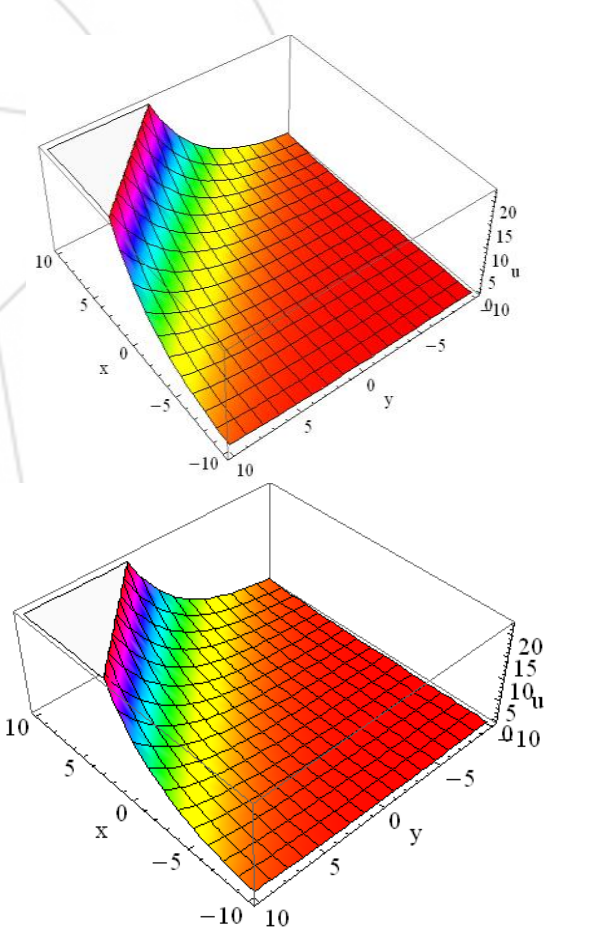

Figure 4: The surface shows the solution $u(x, y, t)$ for (6.17): (i) $\hbar=-1.4, \alpha=0.95$ (ii) $\hbar=-1, \alpha=0.95$ when $h=0.01, t=10, r=48$

Example3. Consider the Eq. (1.1) with $h=1, r=0, a=1$, we have the following fractional biological population equation

$$
\frac{\partial^{\alpha} u}{\partial t^{\alpha}}=\frac{\partial^{2} u^{2}}{\partial x^{2}}+\frac{\partial^{2} u^{2}}{\partial y^{2}}+u
$$

subject to the initial conditions

$$
u_{0}=\sqrt{\operatorname{Sin} x \operatorname{Sinh} y}
$$

now taking the Sumudu transform of both sides of (6.21), and using (6.22) we have

\section{Volume 5 Issue 5, May 2016}

\section{www.ijsr.net}




$$
\begin{gathered}
S[u]-\sqrt{\operatorname{Sin} x \operatorname{Sinh} y}-u^{\alpha}\left[S\left\{\frac{\partial^{2} u^{2}}{\partial x^{2}}+\frac{\partial^{2} u^{2}}{\partial y^{2}}+u\right\}\right]=0 \\
\mathfrak{R}\left(\bar{u}_{m-1}\right)=S\left[u_{m-1}\right]-\left(1-\chi_{m}\right) \sqrt{\operatorname{Sin} x \operatorname{Sinh} y} \\
-u^{\alpha}\left[S\left[\frac{\partial^{2}}{\partial x^{2}} \sum_{j=0}^{m-1} u_{j} u_{m-1-j}\right]+S\left[\frac{\partial^{2}}{\partial y^{2}} \sum_{j=0}^{m-1} u_{j} u_{m-1-j}\right]+S\left[u_{m-1}\right]\right]
\end{gathered}
$$

The $\mathrm{m}^{\text {th }}$ - order deformation is given by

$$
S\left[u_{m}(x, t)-\chi_{m} u_{m-1}(x, t)\right]=\hbar \Re_{m}\left(\vec{u}_{m-1}\right)
$$

Applying the inverse Sumudu transform of above equation, we have

$$
u_{m}(x, t)=\chi_{m} u_{m-1}(x, t)+S^{-1}\left[\hbar \Re_{m}\left(\vec{u}_{m-1}\right)\right]
$$

Solving eq. (6.26) for $\mathrm{m}=1,2,3, \ldots$ we have

$$
\begin{gathered}
u_{1}=-h \hbar \sqrt{\operatorname{Sin} x \operatorname{Sinhy}} \frac{t^{\alpha}}{\Gamma(1+\alpha)} \\
u_{2}=-h \hbar(1+\hbar) \sqrt{\operatorname{Sin} x \operatorname{Sinhy}} \frac{t^{\alpha}}{\Gamma(1+\alpha)}+h^{2} \hbar^{2} \sqrt{\operatorname{Sin} x \operatorname{Sinhy}} \frac{t^{2 \alpha}}{\Gamma(1+2 \alpha)} \\
u_{3}=-h \hbar(1+\hbar)^{2} \sqrt{\operatorname{Sin} x \operatorname{Sinhy}} \frac{t^{\alpha}}{\Gamma(1+\alpha)}+2 h^{2} \hbar^{2}(1+\hbar) \sqrt{\operatorname{Sin} x \operatorname{Sinhy}} \frac{t^{2 \alpha}}{\Gamma(1+2 \alpha)}-h^{3} \hbar^{3} \sqrt{\operatorname{Sin} x \operatorname{Sinhy}} \frac{t^{3 \alpha}}{\Gamma(1+3 \alpha)} \\
u_{4}=-h \hbar(1+\hbar)^{3} \sqrt{\operatorname{Sin} x \operatorname{Sinhy}} \frac{t^{\alpha}}{\Gamma(1+\alpha)}+3 h^{2} \hbar^{2}(1+\hbar)^{2} \sqrt{\operatorname{Sin} x \operatorname{Sinhy}} \frac{t^{2 \alpha}}{\Gamma(1+2 \alpha)} \\
-3 h^{3} \hbar^{3}(1+\hbar) \sqrt{\operatorname{Sin} x \operatorname{Sinhy}} \frac{t^{3 \alpha}}{\Gamma(1+3 \alpha)}+h^{4} \hbar^{4} \sqrt{\operatorname{Sin} x \operatorname{Sinhy}} \frac{t^{4 \alpha}}{\Gamma(1+4 \alpha)}
\end{gathered}
$$

substituting $u_{0}, u_{1}, u_{2}, u_{3}, u_{4}, \ldots$, into Eq. (5.11) gives the solution in series form by, $\hbar=-1$, we have

$$
\begin{gathered}
u(x, y, t)=\sqrt{\operatorname{Sin} x \operatorname{Sinh} y}\left[1+h \frac{t^{\alpha}}{\Gamma(1+\alpha)}+h^{2} \frac{t^{2 \alpha}}{\Gamma(1+2 \alpha)}+h^{3} \frac{t^{3 \alpha}}{\Gamma(1+3 \alpha)}+h^{4} \frac{t^{4 \alpha}}{\Gamma(1+4 \alpha}\right. \\
\text { as } \alpha \rightarrow 1, \text { we have } \\
u(x, y, t)=\sqrt{\operatorname{Sin} x \operatorname{Sinhy}\left[1+h t+\frac{h^{2} t^{2}}{2 !}+\frac{h^{3} t^{3}}{3 !}+\frac{h^{4} t^{4}}{4 !}+\ldots\right](6.28)} \\
u(x, y, t)=\sqrt{\operatorname{Sin} x \operatorname{Sinh} y} e^{t}(6.29) \\
\text { which is an exact solution. }
\end{gathered}
$$

Example4. Consider the Eq. (1.1) with $a=-1, b=1$, we have the following fractional biological population equation

subject to the initial conditions

$$
\frac{\partial^{\alpha} u}{\partial t^{\alpha}}=\frac{\partial^{2} u^{2}}{\partial x^{2}}+\frac{\partial^{2} u^{2}}{\partial y^{2}}+h u^{-1}-h r
$$

$$
u_{0}=\sqrt{\frac{h r}{4} x^{2}+\frac{h r}{4} y^{2}+y+5}
$$

now taking the Sumudu transform of both sides of (26), and using (27) we have

$$
\begin{aligned}
S[u]- & \sqrt{\frac{h r}{4} x^{2}+\frac{h r}{4} y^{2}+y+5}-u^{\alpha}\left[S\left[\frac{\partial^{2} u^{2}}{\partial x^{2}}+\frac{\partial^{2} u^{2}}{\partial y^{2}}+\left(\frac{h}{u_{m-1}}-h r\right)\right]\right]=0 \\
\mathfrak{R}\left(\bar{u}_{m-1}\right)= & S\left[u_{m-1}\right]-\left(1-\chi_{m}\right) \sqrt{\frac{h r}{4} x^{2}+\frac{h r}{4} y^{2}+y+5} \\
& -u^{\alpha}\left[S\left[\frac{\partial^{2}}{\partial x^{2}} \sum_{j=0}^{m-1} u_{j} u_{m-1-j}\right]+S\left[\frac{\partial^{2}}{\partial y^{2}} \sum_{j=0}^{m-1} u_{j} u_{m-1-j}\right]+S\left[\frac{h}{u_{m-1}}-h r\right]\right]
\end{aligned}
$$

The $\mathrm{m}^{\text {th }}$ - order deformation is given by

\section{Volume 5 Issue 5, May 2016}




\section{International Journal of Science and Research (IJSR) \\ ISSN (Online): 2319-7064}

Index Copernicus Value (2013): 6.14 | Impact Factor (2015): 6.391

$$
S\left[u_{m}(x, t)-\chi_{m} u_{m-1}(x, t)\right]=\hbar \Re_{m}\left(\vec{u}_{m-1}\right)
$$

Applying the inverse Sumudu transform of above equation, we have

$$
u_{m}(x, t)=\chi_{m} u_{m-1}(x, t)+S^{-1}\left[\hbar \Re_{m}\left(\vec{u}_{m-1}\right)\right]
$$

Solving eq. (6.35) for $\mathrm{m}=1,2,3, \ldots$, we have

$$
\begin{gathered}
u_{1}=-h \hbar \sqrt{\frac{h r}{4} x^{2}+\frac{h r}{4} y^{2}+y+5} \frac{t^{\alpha}}{\Gamma(1+\alpha)} \\
u_{2}=-h \hbar(1+\hbar) \sqrt{\frac{h r}{4} x^{2}+\frac{h r}{4} y^{2}+y+5} \frac{t^{\alpha}}{\Gamma(1+\alpha)}+h^{2} \hbar^{2}\left(\frac{h r}{4} x^{2}+\frac{h r}{4} y^{2}+y+5\right)^{-1 / 2} \frac{t^{2 \alpha}}{\Gamma(1+2 \alpha)} \\
u_{3}=-h \hbar(1+\hbar)^{2} \sqrt{\frac{h r}{4} x^{2}+\frac{h r}{4} y^{2}+y+5} \frac{t^{\alpha}}{\Gamma(1+\alpha)}+2 h^{2} \hbar^{2}(1+\hbar)\left(\frac{h r}{4} x^{2}+\frac{h r}{4} y^{2}+y+5\right)^{-1 / 2} \frac{t^{2 \alpha}}{\Gamma(1+2 \alpha)}- \\
h^{3} \hbar^{3}\left(\frac{h r}{4} x^{2}+\frac{h r}{4} y^{2}+y+5\right)^{-3 / 2} \frac{t^{3 \alpha}}{\Gamma(1+3 \alpha)}
\end{gathered}
$$

substituting $u_{0}, u_{1}, u_{2}, u_{3}, \ldots$, into Eq. (5.11) gives the solution in series form by, $\hbar=-1$, Then the approximate solution in series form is

$$
u(x, y, t)=u_{0}+\frac{h t^{\alpha}}{u_{0}} \sum_{n=0}^{\infty} \frac{n+1}{\Gamma(1+(n+1) \alpha}\left(\frac{-h t^{\alpha}}{u_{0}^{2}}\right)^{n}
$$

as $\alpha \rightarrow 1$, we have

$$
u(x, y, t)=u_{0}+\left(\frac{h t}{u_{0}}\right) \exp \left(\frac{-h t}{u_{0}^{2}}\right)(6.37)
$$

which is an exact solution of the integer order biological population.
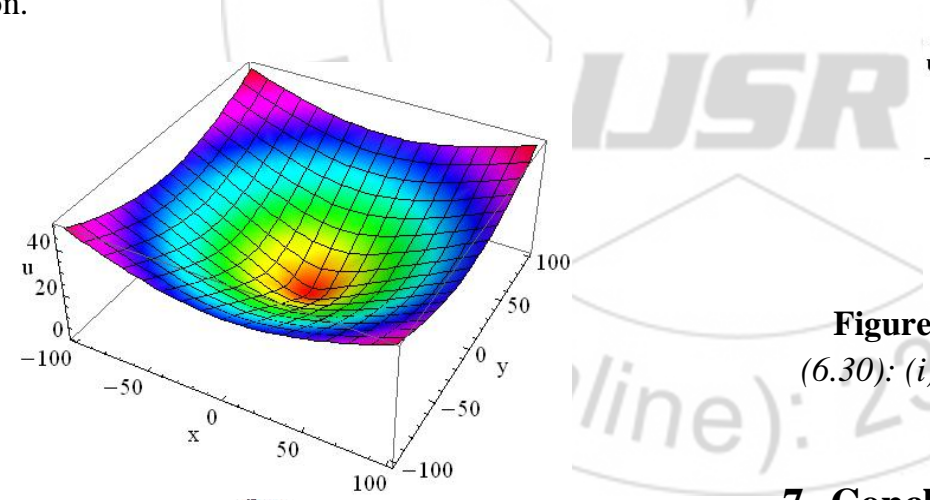

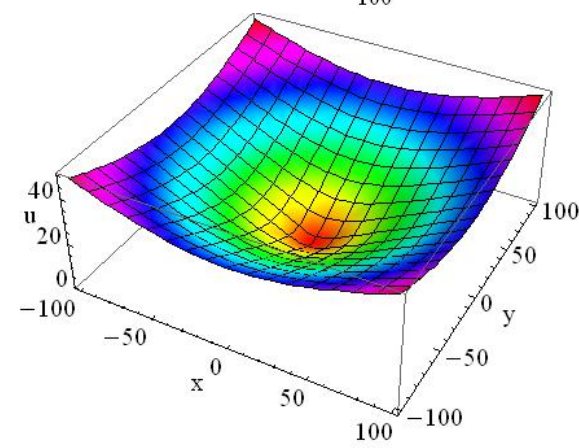

Figure 5: The surface shows the solution $u(x, y, t)$ for (6.30): (i) exact solution (6.37); (ii) numerical solution (6.36) when $h=0.01, t=10, r=48$
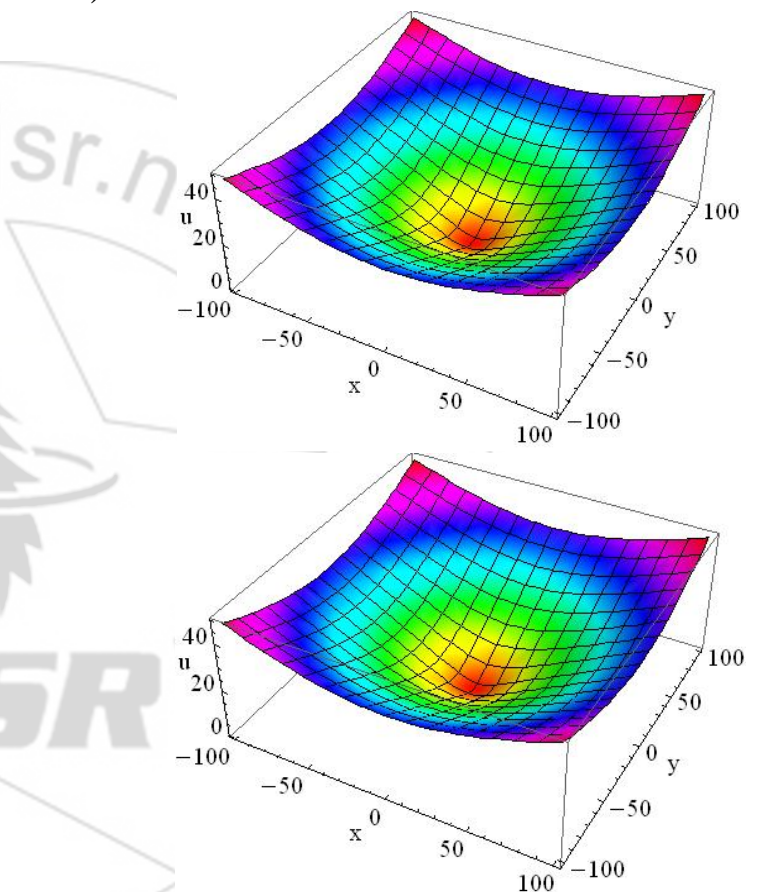

Figure 6: The surface shows the solution $u(x, y, t)$ for (6.30): (i) $\hbar=-1, \alpha=0.9$ (ii) $\hbar=-1, \alpha=0.5$ when

$h=0.01, t=10, r=48$

\section{Conclusion}

We employ the homotopy analysis Sumudu transform method (HASTM) for finding the approximate analytical solutions of time fractional degenerate parabolic equations arising in the spatial diffusion of biological populations subject to the some initial conditions. The results obtained by using this method agree well with the results obtained by ADM [29], VIM [28], HPM [30]. The reliability of HASTM and reduction in computation gives this method a wider applicability. Finally we can conclude that the HASTM is very powerful and efficient in finding analytic as well as numerical solutions for wider classes of linear and nonlinear fractional differential equations. Mathematica has been used for calculation and plot 3D graphs. 


\section{International Journal of Science and Research (IJSR) \\ ISSN (Online): 2319-7064}

Index Copernicus Value (2013): 6.14 | Impact Factor (2015): 6.391

\section{References}

[1] A. Al-rabtah, V.S. Ertürk. Momani, S. Solutions of a fractional oscillator by using differential transform method, Computers and Mathematics with Applications 59(3) (2010), 1356-1362

[2] J.K. Zhou. Differential Transformation and Its Applications for Electrical Circuits, Huazhong University Press, Wuhan, China (1986) in Chinese

[3] G.E. Pukhov. Computational structure for solving differential equations by Taylor transformation, Cybernetics and System Analysis, 14(3) (1978), 383390

[4] H. L. Arora, F.I. Abdelwahid. Solution of non-integer order differential equations via the Adomian decomposition method, Appl. Math. Lett. 6, (1993), 2123

[5] H. Jafari, V. Daftardar-Gejii. Solving linear and nonlinear fractional diffusion and wave equations by Adomian-decomposition. Appl. Math. Comput. 180 (2006), 488-497

[6] S. Momani, A. Al-Khaled. Numerical solutions for system of fractional differential equations by decomposition method. Appl. Math. Comput (196) (2005), 644-665

[7] S. Momani. Analytic approximate solution for fractional heat-like and wave-like equations with variable coefficients using the decomposition method. Appl. Math. Compute. 165 (2005), 459-472

[8] V. Daftardar-Giejji, H. Jafari. Solving a multi order fractional differential equations using Adomian decomposition. J. Math. Anal. Appl. 189 (2007), 5415429.

[9] G.E. Draganescu. Application of a variational iteration method to linear and nonlinear viscoelastic models with fractional derivatives, J. Math. Phys. 47 (8) (2006).

[10] M.R. Yulita, M.S.M. Noorani, I. Hashim. Variational iteration method for fractional heat- and wave - like equations, Nonlinear Analysis: Real World Applications 10 (2009) 1854-1869

[11]Z. Odibat, S. Momani. Application of variation iteration method to non-linear differential equations of fractional order, Int. J. Nonlin. Sci. Numer. Simulat. 1 (7) (2006), $15-27$

[12] J.H. He. Homotopy perturbation technique, Comput. Methods Appl. Mech. Eng. 178 (1999), 257-262.

[13] Q. Wang. Homotopy perturbation method for fractional KdV equation. Appl. Math. Comput. 190 (2007) 17951802

[14]Q. Wang. Homotopy perturbation method for fractional KdV-Burgers equation. Chaos. Soliton. Fractal. 35 (2008), 843-850

[15] S. Momani, Z. Odibat. Homotopy perturbation method for non-linear partial differential equations of fractional order. Phys. Lett. A 365 (5-6), (2007), 345-350

[16]Z. Odibat, S. Momani. Numerical comparison of methods for solving linear differential equations of fractional order. Chaos. Soliton. Fract. 31 (5) (2007), 1248-1255

[17] V.G. Gupta, S. Gupta. Applications of homotopy perturbation transform method for solving heat like and wave like equations with variable coefficients.
International journal of mathematical Archive-2(9) (2011),1582-1590

[18] Y. Khan, Q. B. Wu. Homotopy perturbation transform method for non-linear equations using He's polynomial, Computers and Mathematics with Applications, 61(2011), 1963-1967

[19] Y. Bourermel. Explicit Series Solution for the Glauertjet problem by means of the homotopy analysis method, Int. J. Non-linear Sci. Numer. Simulat. 12 (5) (2007), 714-724

[20] S. J. Liao." An approximate solution technique not depending on small parameters: a special example". International journal of Non-linear mechanics, 30(3) (1995),371-380

[21] S.J. Liao. "A kind of approximate solution technique which does not depend upon small parameters-II. An application in fluid mechanics". International journal of Non-linear mechanics, 32(5) (1997), 815-822

[22] S.J Liao, The Proposed Homotopy Analysis techniques for the Solution of Nonlinear problems, Ph.D. Thesis, Shanghai Jiao Tong University, (1992).

[23] S. J. Liao. Beyond Perturbation: Introduction to homotopy analysis method. CRC Press/ Champan \& Hall, Boca Raton (2003).

[24] S. J. Liao, On Homotopy Analysis Method for nonlinear problems, Appl. Math. Comput. 147 (2004), 499-513

[25] Y.Y. Wu, S. J. Liao. Solving the one loop soliton solution of the Vakhnenko equation by means of the homotopy analysis method. Chaos. Soliton. Fract. 23(5) (2004), 1733-1740

[26] J. Cang, Y. Tan, H. Xu, S. Liao. Series solution of non-linear Riccati differential equations with fractional order, Chaos Soliton. Fract. 40 (1) (2007), 1-9

[27]L. Song, H. Zhang. Application of homotopy analysis method to fractional KdV-Burgers-Kuromoto equation, Phys. Lett. A 367 (1-2) (2007), 88-94

[28]E. Shakeri, M. Dehghan, Numerical solutions of a biological population model using He's variational iteration method, Comput. And maths. with Appl. 54(2007), 1197-1207

[29] S.Z. Rida, A.A.M. Arafa, Exact solutions of fractionalorder biological population model, Commun. Theor. Phys.,52 (2009), 992-996

[30] Y. Liu, Z. Li, Y. Zhang, Homotopy Perturbation method to fractional biological population equation, Fractional Differential Calculus , 1 (1)(2011), 117-124

[31] M. Caputo. Linear models of dissipation whose $Q$ is almost frequency independent. Part III, J. Roy. Astr. Soc. 13(1967), 529-539

[32] G. K. Watugala, Sumudu transform- a new integral transform to solve differential equations and control engineering problems, Math. Engg. Indust. 6 (1998), 319-329

[33] A. Kilicman, H. Eltayeb, A note on integral transforms and partial differential equations, Applied Mathematical Sciences, 4(1-4) (2010), 109-118

[34] M.A. Asiru, Sumudu transform and the solution of integral equation of convolution type, International Journal of Mathematical Education in Science and technology, 32(2001), 906-910.

[35]F.B.M. Belgacem, A.A. karaballi, Sumudu transform fundamental properties investigations and applications, International J. Appl. Math. Stoch. Anal. (2005), 1-23 


\section{International Journal of Science and Research (IJSR) \\ ISSN (Online): 2319-7064}

Index Copernicus Value (2013): 6.14 | Impact Factor (2015): 6.391

[36]F.B.M. Belgacem, A.A. karaballi, S.L. Kalla, Analytical investigations of the Sumudu transform and applications to integral production equations, Mathematical problems in Engineering, 3(2003), 103-118.

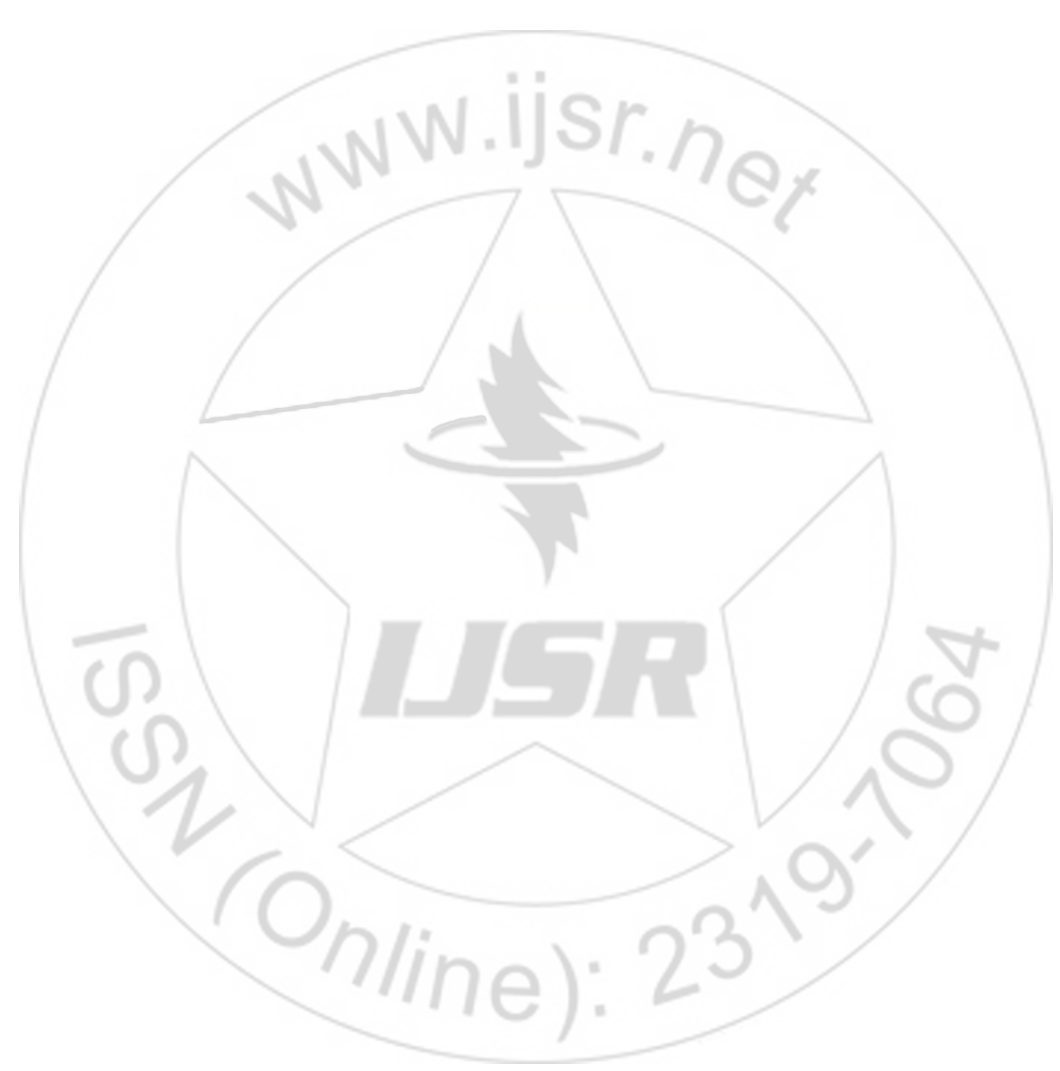

Volume 5 Issue 5, May 2016 